\title{
Isolation and Characterization of Rabbit Gut Transmitted Saccharomyces cerevisiae as a Synbiotic
}

\author{
R. Elanthamil ${ }^{1 *}$, C. Bandeswaran ${ }^{2}$, P. Tensingh Gnanaraj ${ }^{3}$ and K. Vijayarani ${ }^{4}$ \\ ${ }^{1}$ Department of Animal Nutrition, Madras Veterinary College, Chennai, India \\ ${ }^{2}$ Department of Animal Nutrition, Veterinary College and Research Institute, \\ Orthanadu, India \\ ${ }^{3}$ University Research Farm, Madavaram Milk Colony, India \\ ${ }^{4}$ Department of Animal Biotechnology, Madras Veterinary College, Tamil Nadu Veterinary \\ and Animal Sciences University, Chennai-600 051, India \\ *Corresponding author
}

\section{A B S T R A C T}

The use of yeast culture as a dietary supplement has been suggested as a useful tool to stabilize ruminal fermentation. A study was conducted to explore the scope of rabbit gut transmitted (RGT) S. cerevisiae as probiotic and prebiotic (Synbiotic) by in vitro. The rabbit gut transmitted $S$. cerevisiae was isolated from the faeces of rabbits as follows.

Keywords

Saccharomyces

cerevisiae, RGT

Saccharomyces

cerevisiae, Rabbit,

Probiotic, Prebiotic

MOS

Article Info

Accepted:

07 February 2018

Available Online:

10 March 2018 Procured Saccharomyces cerevisiae was propagated, freeze dried and supplemented to adult male New Zealand White rabbits $(4 \times 4)$ at various doses viz., $0.0 \times 10^{8}, 1.5 \times 10^{8}, 3$ $\mathrm{x} 10^{8}, 6 \times 10^{8} \mathrm{CFU} / \mathrm{head} / \mathrm{day}$ for a period of 15 days. Significantly $(\mathrm{P}<0.01)$ highest Saccharomyces cerevisiae excretion in hard faeces of rabbits was observed at highest supplemental dose of Saccharomyces cerevisiae $\left(3 \times 10^{8} \mathrm{CFU} / \mathrm{head} / \mathrm{day}\right)$ at $15^{\text {th }}$ day post supplementation. Faecal isolate of Saccharomyces cerevisiae was confirmed for its origin (supplemental Saccharomyces cerevisiae) through morphological, biochemical assay. The faecal isolate was referred to as "RGT Saccharomyces cerevisiae". Both Saccharomyces cerevisiae and RGT Saccharomyces cerevisiae were assessed for their probiotic and prebiotic characters in seven replications. Probiotic characterization viz., bile tolerance test and $\mathrm{pH}$ tolerance test showed that RGT Saccharomyces cerevisiae had significantly $(\mathrm{P}<0.01)$ higher bile tolerance $(0.3,0.6$ and 0.9 per cent bile) and $\mathrm{pH}$ tolerance $(\mathrm{pH} 2)$ than Saccharomyces cerevisiae. Prebiotic characterization results showed that the MOS ( 0.5 per cent and 1.5 per cent) extracted from RGT Saccharomyces cerevisiae significantly $(\mathrm{P}<0.01)$ improved Lactobacillus acidophilus growth. MOS derived from both Saccharomyces cerevisiae and RGT Saccharomyces cerevisiae, at all level of supplementation significantly $(\mathrm{P}<0.01)$ decreased the Escherichia coli growth.

\section{Introduction}

India has approximately 512 million livestock $\left(19^{\text {th }}\right.$ Livestock census, Government of India,
2012) which are providing livelihood securities to 100 million households distributed in around 6 lakhs villages. India possesses about 15 per cent of the world 
livestock population with only 2 per cent of world geographical area (Singh et al., 2011). Of the total livestock population, about 58.51 per cent are cattle and buffaloes and 39.11 per cent are sheep and goat. Feeding fibrous diets to ruminants is generally practiced in India, which results in lowers productivity. So, there is an urgent need to develop appropriate strategies to manipulate the ruminal fermentation to increase productivity. Some of the feeding strategies like use of prebiotics, probiotics, ionophores, essential oils, flavanoids etc., were emphasized for manipulation. Interest in the use of natural products like probiotics (Lactobacillus spp., Propionibacterium spp., Saccharomyces spp., Bifidiobacterium spp., etc.) as feed additives for ruminant livestock has increased, since the use of antibiotics as growth promoters was banned.

Among the many probiotics, yeast cultures mainly from strains of Saccharomyces cerevisiae are widely used in ruminants to change ruminal fermentation parameters and to have beneficial effects on animal production. Moreover, yeast cells contain different vitamins, enzymes and some unidentified cofactors that may improve the microbial activity and alter the ruminal fermentation pattern. Selections of $S$. cerevisiae strains for to improve the ruminal fiber degradation and overall feed conversion efficiency (Chung et al., 2011).

To prolong the viability of aerobic organism like $S$. cerevisiae in rumen, which is an anaerobic chamber, it was hypothesized that the $S$. cerevisiae which passed through the entire digestive tract of hind gut fermenter like rabbits will have more probiotic efficacy and stability in rumen than other probiotics. Hence, the aim of the present study was designed to assess the efficacy of rabbit gut transmitted $S$. cerevisiae by comparing with standard S. cerevisiae.

\section{Materials and Methods}

This study required no clearance from Ethical committee as major component of the study was in vitro and the feeding trial in rabbits were carried out using surplus bunnies reared for meat in rabbit section of University Research Farm, Madhavaram milk colony, Tamil Nadu Veterinary and Animal Sciences University, Tamil Nadu, India. However the protocol of the experiment, which was a part of M.V.Sc., dissertation of the first author, was reviewed by the programme of research work approval committee, Tamil Nadu Veterinary and Animal Sciences University, Tamil Nadu, India.

\section{Production of rabbit gut transmitted $S$. cerevisiae}

The $S$. cerevisiae culture (MTCC No.4808) was procured from National Institute Multiple Type Cell Culture Collection and Gene Bank, Chandigarh, India. Feeding of $S$. cerevisiae to rabbits and collection of rabbit faeces to isolate RGT $S$. cerevisiae was carried out at University Research Farm, Madhavaram milk colony, Chennai-51. A total of sixteen adult male New Zealand White rabbits were distributed randomly to four treatment groups, each group having four rabbits. The experiment was conducted for fifteen days. The details of feeding regimen followed in the experiment groups are I. Control (Basal diet only) II. Treatment 1 (Basal diet + S.cerevisiae $-1.5 \times 10^{8}$ CFU), III. Treatment 2 (Basal diet $+S$. cerevisiae $-3 \times 10^{8} \mathrm{CFU}$ ) and IV. Treatment 3 (Basal diet $+S$. cerevisiae -6 x $\left.10^{8} \mathrm{CFU}\right)$. The basal diet consisted of adlibitum green fodder (Desmanthus virgatus) and $100 \mathrm{~g}$ of concentrate mixture (maize -60 $\%$, wheat bran $-5 \%$, de oiled rice bran- $7 \%$, soya bean meal $-20 \%$, sun flower oil cake $6 \%$, mineral mixture $-1 \%$ and salt $-1 \%$ ) having crude protein 18 per cent and metabolisable energy $2700 \mathrm{kcal}$ per $\mathrm{kg}$. 
Feeding of $S$. cerevisiae was done by mixing the respective dose of $S$. cerevisiae in the concentrate mixture. Feeding of $S$. cerevisiae was carried out for two weeks period. Hard faeces from the rabbits were collected on $2^{\text {nd }}$, $5^{\text {th }}, 10^{\text {th }}$ and $15^{\text {th }}$ day of the experiment. From the total quantity of hard faeces excreted by the each rabbit, $10 \mathrm{~g}$ (in duplicate) were sampled and used for the isolation of $S$. cerevisiae.

\section{Isolation and characterization}

For isolation of $S$. cerevisiae from the faeces of rabbits, the protocol followed by Kimse et al., (2012) was adopted. The faecal samples were hydrated by adding $90 \mathrm{ml}$ of water to 10 $\mathrm{g}$ of faeces and incubated at $37^{\circ} \mathrm{C}$ for 30 minutes. From the hydrated and incubated faecal samples, serial dilutions were made from $10^{-2}$ to $10^{-4}$. One $\mathrm{ml}$ of the serially diluted faecal samples was loaded on to petri dish containing sterilized agar medium (Sabouraud dextrose agar). Petri dishes were incubated at $30^{\circ} \mathrm{C}$ for 48 hours. Colonies suspected to be yeast were subjected to phenotypic characterization and biochemical tests (carbon source fermentation and nitrogen source utilization) as per methods of Barnett et al., (1990), to ascertain it as yeast ( $S$. cerevisiae) tentatively.

Testing the comparative probiotic and prebiotic efficacy of Saccharomyces cerevisiae and Rabbit gut transmitted Saccharomyces cerevisiae

\section{Probiotic characterization}

Probiotic characterization for its ability to endure fermentation under partial anaerobic condition was tested in the previous stage of the experiment viz., production of rabbit gut transmitted S. cerevisiae. Further screening for probiotic characteristics such as bile tolerance and $\mathrm{pH}$ tolerance was carried out as follows.

\section{Bile and $\mathrm{pH}$ tolerance test}

Sabouraud dextrose broth was supplemented with different concentrations of ox bile salt (0.3, 0.6 and 0.9 per cent) and were inoculated with 1.0 per cent inoculum of $S$. cerevisiae and RGT $S$. cerevisiae and incubated at $30^{\circ} \mathrm{C}$ for 48 hours. After 48 hours of incubation, samples were drawn, serially diluted, plated in Sabouraud dextrose agar plates and incubated at $30^{\circ} \mathrm{C}$ for 48 hours. Growth on plates indicated their tolerance to bile acids (Shukla et al., 2010). Sabouraud dextrose broth with different $\mathrm{pH}(2.0,4.0,6.0$ and 8.0) was adjusted using $1 \mathrm{~N} \mathrm{NaOH}$ or $1 \mathrm{~N} \mathrm{HCl}$. One per cent of $S$. cerevisiae and RGT $S$. cerevisiae was inoculated in different $\mathrm{pH}$ and grown for 48 hours in Sabouraud dextrose broth and colonies were counted in Sabouraud dextrose agar plates by pour plate technique (Shukla $e t$ al., 2010).

\section{Prebiotic characterization}

S. cerevisiae and RGT S. cerevisiae were screened for their prebiotic characteristics. Mannan oligosaccharides (MOS) from both types of $S$. cerevisiae were extracted by bulk culturing, autolyzing and centrifuging. The pellet was freeze dried in a lyophilizer at standard condition (Sutherland and Wilkinson, 1971).

In vitro experiment to evaluate the efficacy of MOS for its impact on selected microorganisms in pour plate technique

In order to assess the extracted MOS for its prebiotic ability, in vitro growth of Lactobacillus acidophilus and Escherichia coli in the presence of the extracted MOS was studied. Lactobacillus acidophilus was maintained in d-MRS broth at $37^{\circ} \mathrm{C}$ under anaerobiosis. Escherichia coli were maintained in nutrient broth at $37^{\circ} \mathrm{C}$. The MOS from $S$. cerevisiae and RGT $S$. 
cerevisiae were tested as substrate at graded level in the media prepared for both microbes viz., d-MRS agar for Lactobacillus acidophilus and MacConkey agar for E. coli. The test was carried out with seven replicates each. The respective media were prepared in such a way that they contained different levels of $\operatorname{MOS}(0.5,1.0,1.5$ and 2.0 per cent) and autoclaved at $121^{\circ} \mathrm{C}$ for 15 minutes. The respective selective media without addition of any MOS served as control. The pour plate procedure was carried out as per Quinn et al., (1992).

\section{Statistical analysis}

The data collected on various parameters were grouped and subjected to statistical analysis by one way ANOVA as per the procedure of statistical analysis system (SPSS, version 20.0 for windows).

\section{Results and Discussion}

Rabbit gut transmitted Saccharomyces cerevisiae production

The faecal concentration of $S$. cerevisiae at different time period ( $\log _{10} \mathrm{CFU} / \mathrm{g}$ ) in hard faeces of adult New Zealand White rabbits fed graded doses of $S$. cerevisiae in their diet is presented in Table 1. From the result, it could be inferred that in all the days of faeces collection $\left(2,5,10\right.$ and $15^{\text {th }}$ day) $S$. cerevisiae $\mathrm{CFU}$ in faeces increased with increase in supplemental dose. Significantly $(\mathrm{P}<0.01)$ highest $S$. cerevisiae excretion in faeces was observed in $\mathrm{T}_{3}$ group of rabbits that were fed the highest supplemental dose of $S$. cerevisiae. Though there was numerical increase in $S$. cerevisiae excretion in $\mathrm{T}_{2}$ group rabbits than $\mathrm{T}_{1}$, statistically it was not significant. Irrespective of the supplemental dose of $S$. cerevisiae fed to rabbits, the $S$. cerevisiae excreted in the faeces significantly $(\mathrm{P}<0.01)$ increased with collection interval viz., $15^{\text {th }}$ day in all treatment groups. It also could be inferred that in all days of faecal collection, there was no $S$. cerevisiae detected in faeces of un-supplemented group (control).Cutting across treatments and days of collection, significantly highest $S$. cerevisiae was excreted in rabbits of $\mathrm{T}_{3}\left(6 \times 10^{8} \mathrm{CFU}\right)$ on $15^{\text {th }}$ day of faecal collection.

\section{Isolation and characterization}

Morphological structure of the colonies suspected to be $S$. cerevisiae appeared as creamy white, round with entire margins, smooth in texture and were elevated in appearance (Plate I). Microscopic appearance (cell morphology) of the suspected colonies of $S$. cerevisiae stained with lacto phenol cotton blue had elliptical or egg shape with some budding cells resembling "Y" (Plate II). The results of the carbon source fermentation and nitrogen source utilization test for $S$. cerevisiae and RGT $S$. cerevisiae is depicted in Table 2 which reveals that $S$. cerevisiae could ferment the carbon source arabinose, fructose, galactose, maltose, rhamnose, sucrose, mannose, trehalose, salicin and glucose but were unable to ferment cellobiose, lactose, mellibiose, raffinose, sorbitol, xylose and mannitol. Similar carbon fermentation characters were also observed in RGT $S$. cerevisiae. Both $S$. cerevisiae and RGT $S$. cerevisiae were capable of utilizing nitrogen from all three sources such as ammonium sulphate, potassium nitrate and urea (Table 2).

Testing the comparative probiotic and prebiotic efficacy of Saccharomyces cerevisiae and Rabbit gut transmitted Saccharomyces cerevisiae

\section{Bile and pH tolerance test}

Table 3 and 4 shows result of growth of $S$. cerevisiae and RGT $S$. cerevisiae on various bile concentrations and different $\mathrm{pH}$ 
respectively. The results confess that both $S$. cerevisiae and RGT $S$. cerevisiae growth significantly declined with increase in bile concentration. Significantly $(\mathrm{P}<0.01)$ highest CFU for both $S$. cerevisiae and RGT $S$. cerevisiae was at zero per cent bile concentration and significantly lowest CFU for both $S$. cerevisiae and RGT S. cerevisiae was at 0.9 per cent bile concentration. On comparing the growth between $S$. cerevisiae and RGT $S$. cerevisiae, it was found that RGT $S$. cerevisiae had a significantly $(\mathrm{P}<0.01)$ higher bile tolerance at all percentage of bile (0.0, 0.3, 0.6 and 0.6 per cent). In different $\mathrm{pH}$, it was evident that both $S$. cerevisiae and RGT $S$. cerevisiae showed significantly $(\mathrm{P}<0.01)$ higher growth in all $\mathrm{pH}$ except at $\mathrm{pH}$ 2. However, the growth between the $\mathrm{pH} \mathrm{4,6}$ and 8 did not significantly differ, though there was a numerical difference in their growth for both $S$. cerevisiae and RGT $S$. cerevisiae While comparing the growth of $S$. cerevisiae with RGT $S$. cerevisiae, it was assert that there was a significantly $(\mathrm{P}<0.01)$ higher growth for RGT $S$. cerevisiae at $\mathrm{pH} 2$ than $S$. cerevisiae

In vitro experiment to evaluate the efficacy of MOS for its impact on selected microorganisms in pour plate technique

The effect of MOS from $S$. cerevisiae and RGT S. cerevisiae on growth of Lactobacillus acidophilus and Escherichia coli is conferred in Table 5 and 6 respectively. The outcome unfold that the survivability of Lactobacillus acidophilus cultured in the medium containing various levels $S$. cerevisiae MOS did not differ significantly though there was numerical variation. But the survivability of Lactobacillus acidophilus cultured in the medium containing RGT $S$. cerevisiae MOS at 1.0 per cent level was significantly $(\mathrm{P}<0.01)$ highest. Further increase in RGT $S$. cerevisiae MOS to 1.5 per cent and 2 per cent caused decline in growth of Lactobacillus acidophilus. On comparing the growth of
Lactobacillus acidophilus in S. cerevisiae MOS and RGT S. cerevisiae MOS medium at 0.5 per cent and 1.0 per cent MOS levels, the survivability of Lactobacillus acidophilus was significantly higher in RGT $S$. cerevisiae MOS medium. At other MOS levels no significant variation was observed in the growth of Lactobacillus acidophilus between $S$. cerevisiae MOS and RGT S. cerevisiae MOS medium. While correlating the growth of Escherichia coli in the medium containing $S$. cerevisiae MOS and RGT $S$. cerevisiae MOS, significantly $(\mathrm{P}<0.01)$ higher growth of Escherichia coli was observed in the medium contains MOS from RGT S. cerevisiae.

S. cerevisiae is an aerobic organism (Dantigny, 1995), its viability in rumen lasts only 24-30 hours (Fonty and ChaucheyrasDurand, 2006). If the viability of S. cerevisiae is to be improved under anaerobic condition, as existing in the rumen, it has to be subjected to similar conditions before it is selected to be used as probiotic in ruminants. Rabbit are hindgut fermenters, the feed in the caecum of rabbit undergoes similar fermentation as that in the foregut of ruminants. Hence, it was hypothesized that the $S$. cerevisiae which passed through the entire digestive tract of rabbit and survived would have more stability and probiotic efficacy for ruminants. Hence this study was designed to produce rabbit gut transmitted $S$. cerevisiae. From the study, it was inferred that excretion of $S$. cerevisiae in hard faeces of rabbit increased with increase in dosage of $S$. cerevisiae and also increased with time of faecal collection after supplementation of $S$. cerevisiae to rabbits. Indicating that various factors affected the survivability of $S$. cerevisiae in the gastrointestinal tract of rabbit and a higher concentration of $S$. cerevisiae supplementation was required because only than a few $S$. cerevisiae could survive the adverse conditions of gastrointestinal tract of rabbit. 
Table.1 Concentration of Saccharomyces cerevisiae at different period of time in hard faeces of adult rabbits fed graded doses of Saccharomyces cerevisiae in the diet (Mean* \pm S.E)

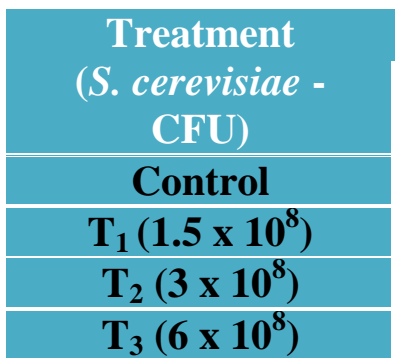

\begin{tabular}{|c|c|c|c|}
\hline $2^{\text {nd }}$ day & $5^{\text {th }}$ day & $10^{\text {th }}$ day & $15^{\text {th }}$ day \\
\hline $0.00^{\mathrm{a}} \pm 0.00$ & $0.00^{\mathrm{a}} \pm 0.00$ & $0.00^{\mathrm{a}} \pm 0.00$ & $0.00^{\mathrm{a}} \pm 0.00$ \\
\hline $0.08^{\mathrm{b} \mathrm{A}} \pm 0.07$ & $4.01^{\mathrm{bB}} \pm 0.26$ & $4.29^{b c} \pm 0.01$ & $4.67^{\mathrm{b} D} \pm 0.01$ \\
\hline $0.19^{\mathrm{b} \mathrm{A}} \pm 0.12$ & $4.05^{\mathrm{b} B} \pm 0.02$ & $4.30^{\mathrm{bC}} \pm 0.01$ & $4.68^{\mathrm{bD}} \pm 0.01$ \\
\hline $0.63^{\mathrm{c} A} \pm 0.02$ & $4.19^{\mathrm{cB}} \pm 0.01$ & $4.48^{\mathrm{c} C} \pm 0.01$ & $4.83^{c D} \pm 0.00$ \\
\hline
\end{tabular}

*Mean of eight observations

Means bearing different superscripts $a, b$ and $c$ in a column differ significantly $(\mathrm{P}<0.01)$

Means bearing different superscripts $A, B, C$ and $D$ in a row differ significantly $(\mathrm{P}<0.01)$

Table. 2 Carbon source fermentation and nitrogen source utilization test for Saccharomyces cerevisiae and RGT Saccharomyces cerevisiae

\begin{tabular}{|c|c|c|}
\hline \multirow[t]{2}{*}{ Test } & \multicolumn{2}{|c|}{ Result } \\
\hline & S. cerevisiae & RGT S. cerevisiae \\
\hline \multicolumn{3}{|l|}{ Carbon source } \\
\hline Arabinose & + & + \\
\hline Cellobiose & - & - \\
\hline Fructose & + & + \\
\hline Galactose & + & + \\
\hline Lactose & - & - \\
\hline Maltose & + & + \\
\hline Mellibiose & - & - \\
\hline Raffinose & + & + \\
\hline Rhamnose & + & + \\
\hline Sorbitol & + & + \\
\hline Sucrose & + & + \\
\hline Xylose & - & - \\
\hline Mannose & + & + \\
\hline Trehalose & + & + \\
\hline Salicin & + & + \\
\hline Mannitol & - & - \\
\hline Glucose & + & + \\
\hline \multicolumn{3}{|l|}{ Nitrogen source } \\
\hline Ammonium sulfate & + & + \\
\hline Potassium nitrate & + & + \\
\hline Urea & + & + \\
\hline
\end{tabular}


Table.3 Effect of various bile concentration on the growth of Saccharomyces cerevisiae and RGT Saccharomyces cerevisiae incubated in selective media for 48 hours in pour plate technique (Mean* \pm S.E)

Bile concentration in
the media $(\%)$
0.0
0.3
0.6
0.9

\begin{tabular}{|c|c|}
\hline $\begin{array}{c}\text { S. cerevisiae } \\
\left(\log _{10} \mathrm{CFU} / \mathrm{ml}\right)\end{array}$ & $\begin{array}{c}\text { RGT S. cerevisiae } \\
\left(\log _{10} \mathrm{CFU} / \mathrm{ml}\right)\end{array}$ \\
\hline $6.04^{\mathrm{d} \mathrm{A}} \pm 0.008$ & $6.14^{\mathrm{d} \mathrm{B}} \pm 0.005$ \\
\hline $5.97^{\mathrm{c} A} \pm 0.000$ & $5.99^{\mathrm{c} B} \pm 0.002$ \\
\hline $5.86^{\mathrm{b} \mathrm{A}} \pm 0.006$ & $5.90^{\mathrm{b} B} \pm 0.003$ \\
\hline $5.62^{\mathrm{a}} \pm 0.004$ & $5.80^{\mathrm{a}} \pm 0.002$ \\
\hline
\end{tabular}

*Mean of seven observations

Means bearing different superscripts $\mathrm{a}, \mathrm{b}, \mathrm{c}$ and $\mathrm{d}$ in a column differ significantly $(\mathrm{P}<0.01)$ Means bearing different superscripts $\mathrm{A}$ and $\mathrm{B}$ in a row differ significantly $(\mathrm{P}<0.01)$

Table.4 Effect of different $\mathrm{pH}$ on the growth of Saccharomyces cerevisiae and RGT Saccharomyces cerevisiae incubated in selective media for 48 hours in pour plate technique (Mean* \pm S.E)

\begin{tabular}{|c|c|c|}
\hline Media pH & $\begin{array}{c}\text { S. cerevisiae } \\
\left(\log _{10} \text { CFU / ml) }\right.\end{array}$ & $\begin{array}{c}\text { RGT S. cerevisiae } \\
\left(\log _{10} \text { CFU / ml) }\right.\end{array}$ \\
\hline 2 & $1.38^{\mathrm{a} A} \pm 0.87$ & $3.48^{\mathrm{a} B} \pm 0.69$ \\
\hline 4 & $6.08^{\mathrm{b}} \pm 0.02$ & $6.12^{\mathrm{b}} \pm 0.01$ \\
\hline 6 & $5.86^{\mathrm{b}} \pm 0.03$ & $5.91^{\mathrm{b}} \pm 0.05$ \\
\hline 8 & $6.02^{\mathrm{b}} \pm 0.01$ & $6.07^{\mathrm{b}} \pm 0.02$ \\
\hline
\end{tabular}

* Mean of seven observations

Means bearing different superscripts a, b, $c$ and $d$ in a column differ significantly $(\mathrm{P}<0.01)$ Means bearing different superscripts $A$ and $B$ in a row differ significantly $(\mathrm{P}<0.01)$

Table.5 Effect of MOS from Saccharomyces cerevisiae and RGT Saccharomyces cerevisiae on the growth of Lactobacillus acidophilus incubated in selective media for 48 hours (Mean* \pm S.E)

\begin{tabular}{|c|c|c|}
\hline \multirow{2}{*}{ MOS (\%) } & \multicolumn{2}{|c|}{ Lactobacillus acidophilus (Log $10 \mathrm{CFU} / \mathrm{ml})$} \\
\hline \multirow{2}{*}{ S. cerevisiae MOS } & RGT S. cerevisiae MOS \\
\hline 0.0 & $6.10 \pm 0.003$ & $6.09^{\mathrm{a}} \pm 0.002$ \\
\hline 0.5 & $6.11^{\mathrm{A}} \pm 0.002$ & $6.13^{\mathrm{b}} \pm 0.003$ \\
\hline 1.0 & $6.18^{\mathrm{A}} \pm 0.002$ & $6.21^{\mathrm{d} B} \pm 0.001$ \\
\hline 1.5 & $6.11 \pm 0.008$ & $6.15^{\mathrm{c}} \pm 0.006$ \\
\hline 2.0 & $6.11 \pm 0.007$ & $6.12^{\mathrm{b}} \pm 0.007$ \\
\hline
\end{tabular}

*Mean of seven observations

Means bearing different superscripts a, $b, c$ and $d$ in column differ significantly $(\mathrm{P}<0.01)$

Means bearing different superscripts $A$ and $B$ in a row differ significantly $(\mathrm{P}<0.01)$ 
Table.6 Effect of MOS from Saccharomyces cerevisiae and RGT Saccharomyces cerevisiae on growth of Escherichia coli incubated in selective media for 48 hours (Mean*士 S.E)

\begin{tabular}{|c|c|c|}
\hline \multirow{2}{*}{ MOS $(\%)$} & \multicolumn{2}{|c|}{ Escherichia coli $\left(\log _{10}\right.$ CFU / ml) } \\
\hline & S. cerevisiae MOS & RGT S. cerevisiae MOS \\
\hline 0.0 & $6.08^{\mathrm{e}} \pm 0.007$ & $6.09^{\mathrm{e}} \pm 0.003$ \\
\hline 0.5 & $5.70^{\mathrm{c}} \pm 0.008$ & $5.71^{\mathrm{c}} \pm 0.005$ \\
\hline 1.0 & $5.62^{\mathrm{b}} \pm 0.010$ & $5.73^{\mathrm{b}} \pm 0.003$ \\
\hline 1.5 & $5.51^{\mathrm{a}} \pm 0.001$ & $5.61^{\mathrm{a}} \mathrm{B} \pm 0.001$ \\
\hline 2.0 & $5.75^{\mathrm{d}} \pm 0.003$ & $5.79^{\mathrm{d}} \mathrm{B} \pm 0.003$ \\
\hline
\end{tabular}

*Mean of seven observations

Means bearing different superscripts a, b, c, d and e in a column differ significantly $(\mathrm{P}<0.01)$

Means bearing different superscripts $\mathrm{A}$ and $\mathrm{B}$ in a row differ significantly $(\mathrm{P}<0.01)$

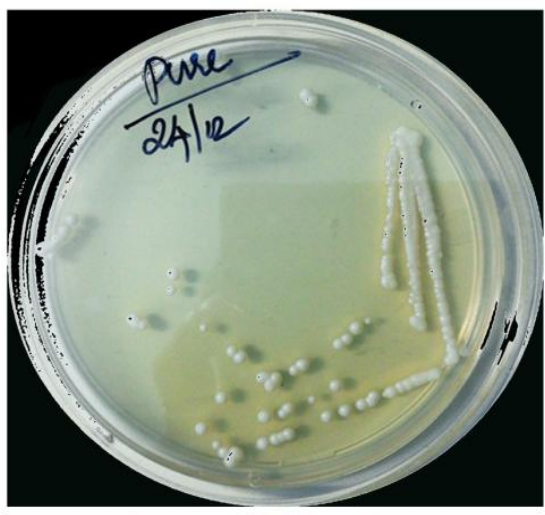

Saccharomyces cerevisiae Plate 1 Colony morphology

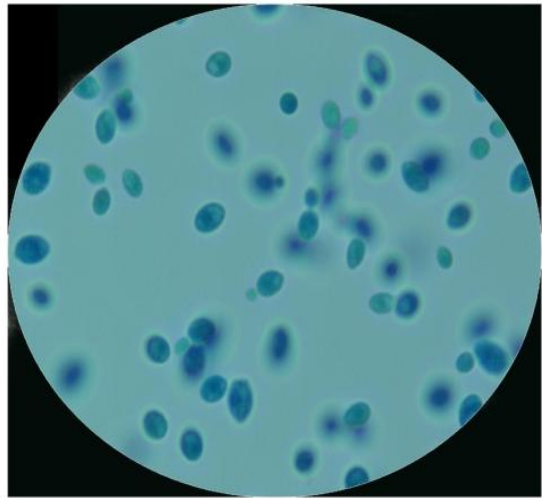

Saccharomyces cerevisiae

Plate 2 Cell morphology

For those $S$. cerevisiae that had survived in the adverse conditions of gastrointestinal tract in rabbit it took 10-15 days for colonization.

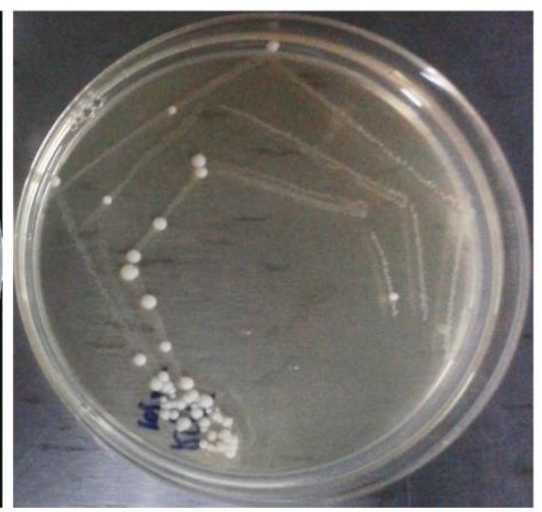

RGT Saccharomyces cerevisiae

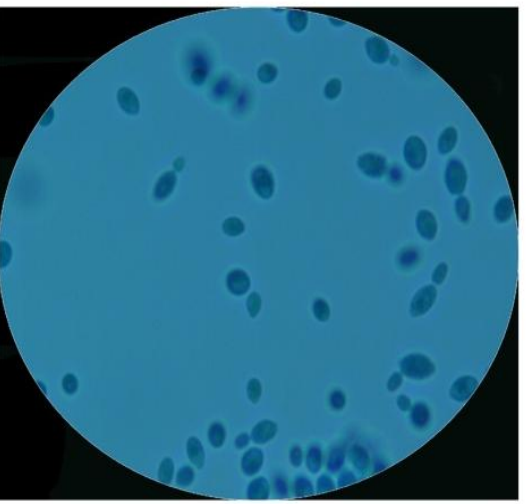

RGT Saccharomyces cerevisiae

This could be the possible reason for the delayed excretion of $S$. cerevisiae in rabbit faeces. 
The organism excreted in the faeces of rabbits that were fed $S$. cerevisiae was identified as $S$. cerevisiae through morphological characterization. Seventy per cent of colonies of $S$. cerevisiae were smooth, white creamy with ovoid or spherical shape (Sulieman et al., 2015). Moreover, S. cerevisiae in its vegetative form is egg shaped, elliptical occasionally spherical (De becze, 1956). Similar observations were documented in this study. Hence the organism isolated from faeces of rabbit was characterized as $S$. cerevisiae phenotypically. All Saccharomyces isolates were capable of fermenting glucose (Sulieman et al., 2015). The capacity of Saccharomyces species to degrade carbon source depends on the enzyme they produce that are necessary for the conversion of sugars to other products (Ebabhi et al., 2013). A wide variety of nitrogen-containing compounds, including ammonium salts, amino acids and di- and tripeptides can be assimilated by $S$. cerevisiae and provide the pools of polyamines, amino acids, nucleotide bases and their derivatives that are required for the production of cell biomass (Crepin et al., 2012). In the present study also $S$. cerevisiae and RGT $S$. cerevisiae were capable of utilizing nitrogen from ammonium sulphate, potassium nitrate and urea for their growth.

In order to complement the taxonomic identification of $S$. cerevisiae and RGT $S$. cerevisiae from morphological and biochemical tests, PCR assays were done using specific primers. The results of the PCR assay revealed that the sequence of both $S$. cerevisiae and RGT $S$. cerevisiae were similar. Thus, confirming the origin of RGT $S$. cerevisiae. Characterization of Saccharomyces isolates is simpler and faster using the gene sequencing method, but needs the inclusion of accurate reference strain for a definite identity (Diosama et al., 2014).
Tolerance to bile salts is considered to be a main prerequisite for growth, colonization and metabolic activity of microorganism in the host's gut (Liong and Shah, 2005). Therefore, it is generally included in the selection criteria of potential probiotic. In this study, both $S$. cerevisiae and RGT $S$. cerevisiae were able to tolerate all levels of bile concentration. RGT $S$. cerevisiae showed better growth than $S$. cerevisiae. However, both the organism showed reduced growth, when there was proportionate increase in bile concentration. The result concurs with the observation of Shukla et al., (2010), who reported that there was a significant decrease in viability of yeast strains with increase in bile concentration. The decreased growth, in increased bile concentration may be attributed to the fact that the probiotic organisms were bound with the bile salts (Patel et al., 2004). Comparison was made between the growth of $S$. cerevisiae and RGT $S$. cerevisiae at various bile concentration across incubation hours and it could be inferred that the growth of RGT $S$. cerevisiae was significantly higher compared to that of $S$. cerevisiae, thus establishing the supremacy of RGT $S$. cerevisiae over $S$. cerevisiae with regard to resistance in bile salt. The difference in the level of bile tolerance may be due to variation in strains (Shukla et al., 2010). Moreover, RGT S. cerevisiae was a strain that had passed through rabbit gut, hence could have developed the capacity for higher bile tolerance.

Most definition of probiotic emphasizes that the microorganisms should be viable and reaches their site of action live (Ouwehand et al., 1999). The primary barrier in the stomach is the inhibitory action of gastric acid, being related to its low $\mathrm{pH}$ (Rajkowska and Kunicka-Styczynska, 2010). In this study, both $S$. cerevisiae and RGT $S$. cerevisiae had $\mathrm{pH}$ tolerance as evinced by their growth at $\mathrm{pH}$ 
2. However, S. cerevisiae at $\mathrm{pH} 2$ showed declined growth after 24 hours of incubation which was not in the case for RGT $S$. cerevisiae, where growth was evident up to 48 hours of incubation at $\mathrm{pH} 2$. The high acid tolerance of RGT $S$. cerevisiae could be attributed to strain variations, some strains are tolerant to acidic conditions than others either due to high cytoplasmic buffering capacity or membrane ATPases, that in turn resists changes in the cytoplasmic $\mathrm{pH}$ and gains stability under acidic conditions (Berada et al., 1991).

Mannan oligosaccharides are complex oligosaccharides derived from the cell wall of $S$. cerevisiae (Spring et al., 2000). The structure of the mannan component of MOS resembles that of the carbohydrates on the intestinal wall. Pathogenic bacteria containing type-I (mannose specific) fimbriae normally adhere to mannan on the mucosal surface of the intestine. The mannan component of MOS provides a competitive binding site for certain intestinal pathogens. Therefore, benefits of MOS are associated with pathogen removal from the intestine without attachment and colonization (Shane, 2001). The current study also demonstrated that increasing level of MOS per cent from both source $S$. cerevisiae and RGT $S$. cerevisiae led to decrease in the growth of E. coli.

Intake of prebiotics can significantly modulate the colonic microbiota by increasing the number of specific probiotic bacteria such as Lactobacilli (Rycroft et al., 2001). Swanson et al., (2002) also reported increased Lactobacillus concentration in ileum following FOS plus MOS supplementation. This study also documented similar results, wherein Lactobacillus count increased on supplemental MOS from both $S$. cerevisiae and $R G T S$. cerevisiae. It is hypothesized that MOS helps to establish a functional ecosystem between the host and the intestinal microflora through intestinal mucins (Uni and Smirnoc, 2006).

\section{References}

Ausubel, F.M., R. Brent, R.E. Kingston, D.D. Moore, J.G. Seidman, J.A. Smith and K. Struhl, 1999. Recombinant DNA technology. Short protocols in molecular biology. $4^{\text {th }}$ edn., Wiley, New York.

Barnett, J.A., R.W. Payne and D. Yarrow, 1990. Yeast characteristic and identification. $2^{\text {nd }}$ edn., Cambridge University press, UK, pp 1-1002.

Berada, N., J.F. Lemeland, G. Laroch, P. Thouvenot and M. Piala, 1991. Bifidobacterium from fermented milks: Survival during gastric transit. Journal of Dairy Science, 74(2): 409- 413.

Chung, Y.H., N.D. Walker, S.M. McGinn and K.A. Beauchemin, 2011. Differing effects of 2 active dried yeast (Saccharomyces cerevisiae) strains on ruminal acidosis and methane production in non-lactating dairy cows. Journal of Dairy Science, 94(5): 24312439.

Crepin, L., T. Nidelet, I. Sanchez, S. Dequin and C. Camarasa, 2012. Sequential use of nitrogen compounds by Saccharomyces cerevisiae during wine fermentation: A model based on kinetic and regulation characteristics of nitrogen permeases. Applied and Environmental Microbiology, 78(22): 8102-8111.

Dantigny, P., 1995. Modeling of the aerobic growth of Saccharomyces cerevisiae on mixtures of glucose and ethanol in continuous culture. Journal of Biotechnology, 43(3): 213-220.

De Becze, G.I., 1956. Yeasts: I. Morphology, A microbiological process report, Applied Microbiology, 4(1): 1-12. 
Diosama, G., D.E. Romanin, M.F. ReyBurusco, A. Londero and G.L. Garrote, 2014. Yeasts from kefir grains: Isolation, identification and probiotic characterization. World Journal of Microbial Biotechnology, 30(1): 43-53.

Ebabhi, A.M., A.A. Adekunle, W.O Okunowo and A.A. Osuntoki, 2013. Isolation and characterization of yeast strains from local food crops. Journal of Yeast and Fungal Research, 4(4): 3843.

Fonty, G. and F. Chaucheyras-Durand, 2006. Effects and mode of action of live yeasts in rumen. Biologia, 61(6): 741750.

Kimsé, M., C. Bayourthe, V. Monteils, L. Fortun-Lamothe, L. Cauquil, S. Combes and T. Gidenne, 2012. Live yeast stability in rabbit digestive tract: Consequences on the caecal ecosystem, digestion, growth and digestive health. Animal Feed Science and Technology, 173: 235-243.

Liong, M. and N.P. Shah, 2005. Acid and bile tolerance and cholesterol removability of lactobacilli strains. Journal of Dairy Science, 88(1): 55-66.

Ouwehand, A.C., P.V. Kirjavainen, C. Shortt and S. Salminen, 1999. Probiotics: Mechanisms and established effects. International Dairy Journal, 9: 43-52.

Patel, H.M., S.S. Pandiella, R.H. Wang and C. Wevb, 2004. Influence of malt, wheat and barley extract on the bile tolerance of selected strain of Lactobacilli. Food Microbiology, 21: 83-59.

Quinn, P.J., M.E. Carter, B.K. Markey and G.R. Carter, 1992. Clinical Veterinary Microbilogy. Mosby-year book Europe Limited, Lynton House, Tavistock square, London, pp 61-65.

Rajkowska, K. and A. Kunicka-Styczynska, 2010. Probiotic properties of yeasts isolated from chicken faeces and kefirs.
Polish Journal of Microbiology, 59(4): 257-263.

Rycroft, C.E., M.R. Jones, G.R. Gibson and R.A. Rastall, 2001. A comparative in vitro evaluation of the fermentation properties of prebiotic oligosaccharides. Journal of Applied Bacteriology, 91(5): 878-887.

Sabate, J., J.M. Guillamon and J. Caro, 2000. PCR differentiation of Saccharomyces cerevisiae from Saccharomyces bayanus/Saccharomyces pastoriamus using specific primers. FEMS Microbiology Letter, 193(2): 255-259.

Shane, S.M., 2001. Mannan oligosaccharides in poultry nutrition, mechanisms and benefits. In: Proceedings of Alletch's $17^{\text {th }}$ Annual Symposium, Nottingham University Press, Nottingham, United Kingdom. pp 65-77.

Shukla, G., G. Sharma and N. Goyal, 2010. Probiotic characterization of Lactobacilli and yeast isolated from whey beverage and therapeutic of Lactobacillus yogurt in murine giardiasis. American Journal of Biomedical Sciences, 2(3): 248-261.

Singh S.V., R.C. Upadyaya and Ashutosh, 2011. Climate change: Impact on animal performance and mitigation Strategies. Indian Dairy Man, 63: 5056.

Spring, P., C. Wenk, K.A. Dawson and K.E. Newman, 2000. The effects of dietary mannanoligosaccharides on cecal parameters and the concentrations of enteric bacteria in the ceaca of Salmonella-challenged broiler chicks. Poultry Science, 79(2): 205-211.

Sulieman, A.M.E., A.M. Esra and W.S. Abdelgadir, 2015. Isolation and identification of yeasts from the different stages of Hulu-mur fermentation. Journal of Microbiology Research, 5(2): 71-76. 
Sutherland, I.W. and J.F. Wilkinson, 1971. Chemical extraction methods of microbial cells. Methods in Microbiology, Vol. 5B, Academic Press, London. pp 346-383.

Swanson, K.S., C.M. Grieshop, E.A. Flickinger, H.P. Healy, K.A. Dawson, N.R. Merchen and G.C. Fahey, 2002. Effects of supplemental fructooligosaccharides plus mannanoligosaccharides on immune function and ileal and fecal microbial populations in adult dogs. Archives of Animal Nutrition, 56(4): 309-318.

Uni, Z. and A. Smirnoc, 2006. Modulating mucin dynamics using functional carbohydrates. Reproduction Nutrition Development, 46(Suppl. 1): S76.

\section{How to cite this article:}

Elanthamil, R., C. Bandeswaran, P. Tensingh Gnanaraj and Vijayarani, K. 2018. Isolation and Characterization of Rabbit Gut Transmitted Saccharomyces cerevisiae as a Synbiotic. Int.J.Curr.Microbiol.App.Sci. 7(03): 871-882. doi: https://doi.org/10.20546/ijcmas.2018.703.102 